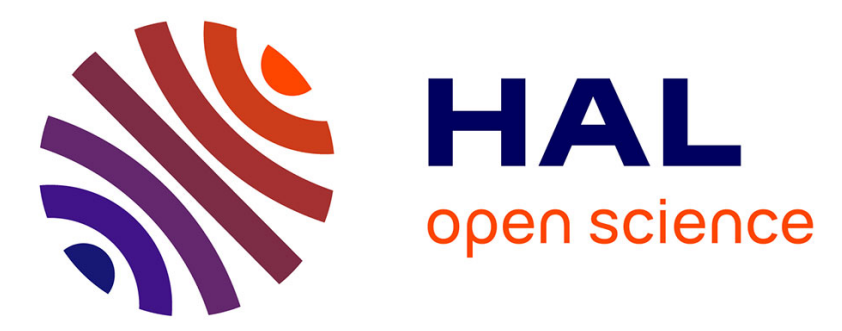

\title{
Molecular analysis for medicine: a new technological platform based on nanopatterning and label-free optical detection
}

Jean Christophe Cau, Hélène Lalo, Childérick Séverac, Jean Pierre Peyrade, Emmanuelle Trevisiol, Véronique Le Berre, J. Francois, Christophe Vieu

\section{To cite this version:}

Jean Christophe Cau, Hélène Lalo, Childérick Séverac, Jean Pierre Peyrade, Emmanuelle Trevisiol, et al.. Molecular analysis for medicine: a new technological platform based on nanopatterning and label-free optical detection. Oncologie, 2009, 11 (S1), pp.148 - 152. 10.1007/s10269-009-1825-7. hal-01849402

\section{HAL Id: hal-01849402 https://hal.science/hal-01849402}

Submitted on 5 Jun 2019

HAL is a multi-disciplinary open access archive for the deposit and dissemination of scientific research documents, whether they are published or not. The documents may come from teaching and research institutions in France or abroad, or from public or private research centers.
L'archive ouverte pluridisciplinaire HAL, est destinée au dépôt et à la diffusion de documents scientifiques de niveau recherche, publiés ou non, émanant des établissements d'enseignement et de recherche français ou étrangers, des laboratoires publics ou privés. 


\title{
Molecular analysis for medicine: a new technological platform based on nanopatterning and label-free optical detection
}

\author{
J.C. Cau ${ }^{1,2}$, H. Lalo ${ }^{1,2}$, C. Séverac ${ }^{1}$, J.P. Peyrade ${ }^{1}$, E. Trévisiol ${ }^{1,3}$, V. Leberre ${ }^{3}$, \\ J.M. Francois ${ }^{3}$, C. Vieu ${ }^{1}$ \\ ${ }^{1}$ LAAS-CNRS, University of Toulouse, 7 avenue du colonel Roche, Toulouse F-31077, France \\ ${ }^{2}$ INNOPSYS Parc d'Activités Activestre, Carbonne F-31390, France \\ ${ }^{3}$ LISBP, CNRS-INRA, University of Toulouse, 135 avenue de rangueil, Toulouse F-31077, France \\ Correspondance : cvieu@laas.fr \\ Reçu le 20 octobre 2009 ; accepté le 28 octobre 2009
}

\section{Une nouvelle plateforme d'analyse moléculaire pour le diagnostic médical basée sur la nanolithographie douce et la biodétection optique sans marquage}

Résumé : Dans ce travail nous montrons que la structuration à l'échelle nanométrique de biomolécules sondes par lithographie douce permet de fabriquer des puces à protéines à un coût de production suffisamment réduit pour entrevoir leur utilisation dans le domaine de l'analyse moléculaire médicale. La combinaison d'un procédé d'impression moléculaire et d'une détection optique sans marquage fondée sur le principe de la diffraction de la lumière est mise en œuvre afin de produire des supports d'analyse en verre comportant des motifs nanométriques et un scanner de diffraction qui permet la lecture d'un test biologique multiplexé.

Mots clés : Biopuces - Nanostructuration - Lithographie douce Biodétection sans marquage - Diagnostic medical - Puces à protéines Tests immunologiques

Abstract: In this article, we show that by biopatterning probe molecules at the nanoscale using soft lithography, protein biochips can be produced at a significantly lower cost for their use as a systematic method of molecular analysis for medical diagnosis purposes. The combination of multiplexed nanoscale microcontact printing and label-free optical detection using the principle of light diffraction is implemented for generating engineered glass slides for analysis, and a dedicated diffractive scanner for reading the multiplexed results of an assay.

Keywords: Biochip - Nanopatterning - Soft lithography - Label-free biosensing - Medical diagnosis Protein chips - Immunoassays

\section{Introduction}

In the field of nanomedicine and more specifically in oncology, nanotechnologies will have a deep impact on drug delivery processes and analytical devices for detecting various kinds of biomarkers, with ultra high sensitivity [4]. After many demonstrations in research laboratory environments confirming these promises, these technologies based on various nanoobjects, such as nanoparticles and nanowires, now face the problem of performance variabilities. Reliability will be a major issue in this field, before some of these innovative devices become standard tools in cancer research. The reliability of a medical diagnosis based on a molecular detection at the Femto molar level remains to be established in real conditions and real samples obtained from patients. In this field, ultra high sensitivity conflicts dramatically with confidence and discriminating capabilities to avoid the multiplication of false positives.

Another potential benefit of nanotechnology for medical diagnosis based on molecular analysis is the possibility to reduce drastically the cost of biochip technology. This improvement would render possible the use of such molecular analysis at the level of genes or proteins for a large number of patients. Here, the bottleneck that is surpassed through nanotechnologies is much more economical than cognitive. In this direction, we have investigated a method for producing biochips (DNA or proteins) at low cost using soft lithography. Moreover, by exploiting the ability of this method to pattern probe molecules at the nanometric level, we have designed a specific pattern enabling to perform the biodetection without any fluorescent labeling. This article explains the principles of soft lithography, label free detection using diffractive nanopatterns, and the validation of this new technology.

In any biochip technology, the aim is to detect a large number of possible specific interactions between probe molecules deposited on the surface at well-registered 
positions and target molecules present in the solution being analyzed at an unknown concentration on a solid support. Similar to the procedure employed for DNA microarrays, through the precise knowledge of the sequences deposited on the matrix of spots, the detection of specific genes expressed in the analyte is rendered possibly through the optical reading of the chip, as soon as the target molecules are labeled with light-emitting fluorophores. This technology [10] is very well established for DNA molecules where hybridization events are detected and has also been developed for mapping proteins [1] using specific antibodies primarily as probe molecules. However, the cost of the method prevents its implementation for routine medical analysis. Today, the detection of multiple cancer biomarkers using protein biochips would probably have a major impact for medical diagnostics, but they are not used because of their prohibitive cost of implementation. Only research activities can benefit from this technology. This high cost is mainly due to the following three aspects: i) the difficulty to deposit multiple probe molecules on a surface (typically a glass slide), which requires either expensive automatic robots [11] or advanced photolithography methods [5]; ii) the requirement of labeling target molecules with fluorophores for enabling fluorescence detection to be performed; and iii) the necessity to use quite large quantities of probe molecules, which can be expensive, due to the micrometric dimensions of the spots on the chip and the limit of detection of fluorescence signals requiring a given quantity of interactions for discriminating the fluorescent signal from the noisy background. A new biochip platform of low cost should therefore combine a low-cost deposition method of multiple biological spots on a glass slide, a label free detection method, and an improved limit of detection. The method we propose in this article depends on the use of soft lithography for the biopatterning process, a new label-free detection method based on the detection of diffracted optical beams, and the generation of nanoscale patterns that enable a drastic reduction of the detection limit compared to fluorescence.

\section{Deposition of multiple probe biomolecules using microcontact printing and macrostamps}

Since its discovery more than a decade ago [7], microcontact printing ( $\mu \mathrm{CP}$ ) has become a well-established technique, capable of patterning in a highly parallel way, a large variety of molecules on arbitrary surfaces for research purposes. Initially, the technique was proposed as a route for creating patterns of self-assembled monolayers (SAMs) $[3,6,7]$ and was later extended to the patterning of biomolecules [2,9]. The transfer of these molecules along well-controlled features is achieved through the use of a patterned elastomeric stamp, typically fabricated from poly(dimethylsiloxane) (PDMS) [15] casted onto a lithographically patterned silicon hard master. PDMS material turned out to possess unique advantages with respect to the printing process, such as its ability to exhibit conformal contact on rough surfaces and its chemical inertness. During $\mu \mathrm{CP}$, the elastomeric stamp is inked with a solution containing the molecules to be deposited and is gently brought into contact with the substrate. Ideally, the molecules of the ink are delivered to the surface only in the contact areas corresponding to the protrusions of the stamp. In brief, the molecules are stamped manually on the surface like any ink deposited using a printing process. The method has proven to be able to pattern molecular features with a spatial resolution at the nanoscale [13], can be implemented with limited equipments (manual printing works perfectly), and relies on very low-cost material (PDMS). Of course, the silicon master used for creating the PDMS elastomeric replica requires advanced lithography methods for being fabricated [14], but a silicon master can be used a very large number of times $\left(>10^{3}\right)$, and each stamp can be regenerated for several successive prints. Recently, our group has demonstrated the possibility to print DNA features for microarray applications using $\mu \mathrm{CP}$ [12]. We have shown that, in opposition to previous publications, the print could be achieved without any surface treatment of the PDMS stamp. The fluorescence signal arising from these printed spots deposited by $\mu \mathrm{CP}$ turned out to be systematically much stronger than the conventional spots deposited by metal pins because of a better organisation of the DNA probes at the chip surface. This advantage enabled us to use significantly smaller concentrations of probe molecules. Similar improvements were also observed for protein chips. In summary, we came rapidly to the conclusion that $\mu \mathrm{CP}$ could be used for high-quality deposition of biomolecules on glass slides for biochip applications. However, the major bottleneck remaining to be solved for real useful applications is that only one type of biomolecule can be printed in one step because, in general, the stamp is inked everywhere with the same biomolecule. We have thus developed and patented a novel method for multiplexing the $\mu \mathrm{CP}$ technique that targets the deposition of 100-1000 different molecules in one step [8]. For this specific purpose, we have fabricated specific hierarchical stamps, called macrostamps, containing millimetric pads arranged in a periodic array compatible with titration plates (Fig. 1). Through a very simple procedure of molding, each of this pad includes micro- and nanoscale features on its surface. Then by inking in a single step each dot with a different ink through a titration plate, it becomes possible to print different inks in one step and to obtain simultaneously nanoscale patterns of many different biomolecules at a time. Our latest results show that without any trace of contamination up to 800 different biological inks can be “nanoprinted' in one step.

The validation of these multilevel stamps has been achieved using fluorescent inks and DNA 


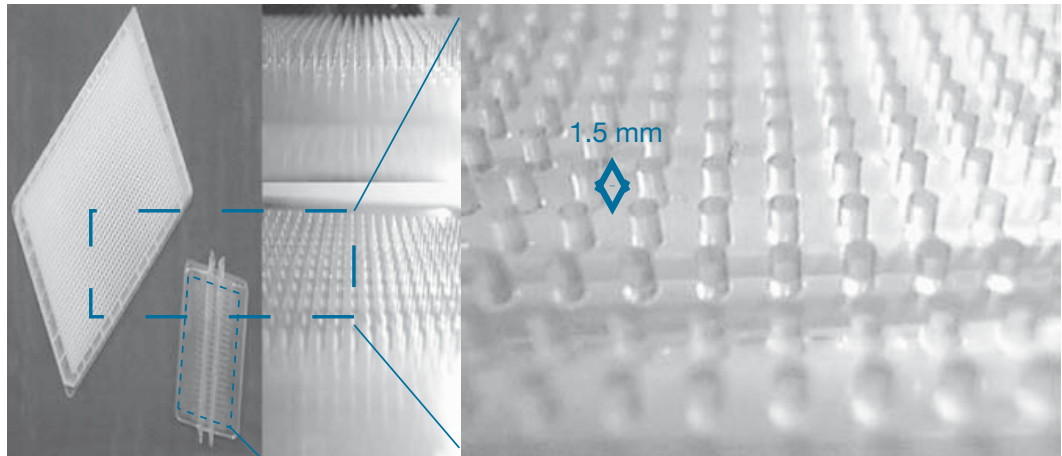

Fig. 1.

Photography of a large multilevel PDMS stamp compliant with the format of a 1536 titration plate. Each of the protruding millimetric pads contains micro/nanoscale features that cannot be seen at this magnification
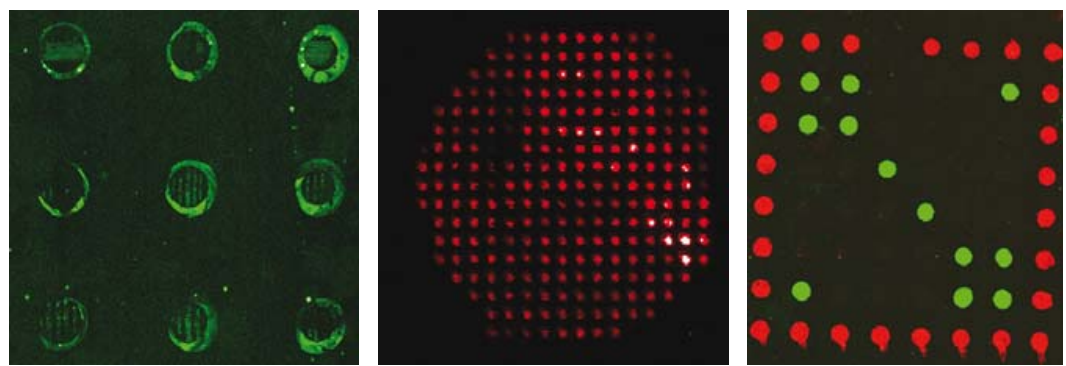

Fig. 2.

Right side: Fluorescence image of nine zones among 200 that were printed in one step. Each zone contains micro and nanoscale features hardly visible at this magnification. Each circular zone has a diameter of $1 \mathrm{~mm}$ and can be inked with a specific ink. Middle: enlarged view of a single zone showing an array of dots of $1 \mu \mathrm{m}$, the pixelization of the image is due to the fluorescence scanner used for the characterization. Left side: green and red fluorophores printed in a single step showing the absence of contamination between adjacent spots after DNA hybridization

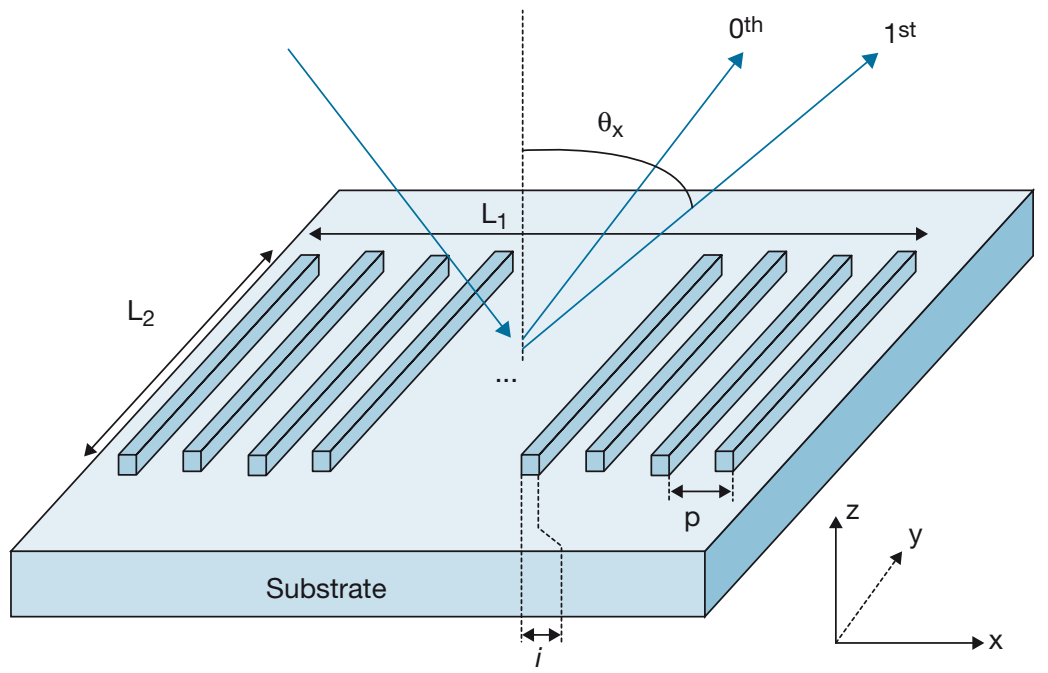

Fig. 3.

Principle of the diffractive label-free biosensing. Probe molecules are printed by $\mu \mathrm{CP}$ into a periodic grating of lines. The period $P$ is in the order of $1 \mu \mathrm{m}$, and the linewidth is submicrometric. An incident laser beam is diffracted efficiently in various orders of diffraction. The first-order beam is used for detecting specific probe/target interactions through a simple measurement of the diffracted intensity probe molecules. The results show that a reliable printing can be obtained over a few hundreds of pads in one step of contact printing and that no trace of contamination can be found from one pad to another. This latter result has been checked by alternating red and green fluorophores from pad to pad and observing the fluorescence of the patterns. Typical results of printing are shown in Figure 2. The presented images have been characterized using a fluorescence scanner for biochip technology, which is limited to a pixel size of $5 \mu \mathrm{m}$. This is the reason why submicronic features cannot be observed in these images.

\section{Diffraction-based biosensing using nanoscale periodic gratings of probe molecules}

Taking benefit of the ability of $\mu \mathrm{CP}$ to pattern biomolecules along nanoscale features of any shape and dimensions, we have investigated a new optical principle of detection that does not rely on any labeling method. Our idea was to arrange the probe molecules into periodic gratings, capable to efficiently diffract incident monochromatic light in various orders of diffraction. Upon specific interactions of these probe biomolecules with target molecules incubated in an analyte, the intensity of the diffracted light is modified and this variation can be used for quantitative label-free biodetection (Fig. 3). This principle is implemented to finally build a scanner that enables the measurement of the diffracted intensity by each array of a full biochip, where several thousands of gratings are generated by multiplexed $\mu \mathrm{CP}$. This so-called diffractive scanner replaces the conventional fluorescence scanner in current biochip technology.

Figure 4 shows an example of a periodic grating of proteins obtained by multiplexed $\mu \mathrm{CP}$ that are used for diffraction-based sensing.

We have validated our detection method using a protocol involving the interaction between protein $A$ and a specific antibody (antiprotein A). In these experiments of 
A
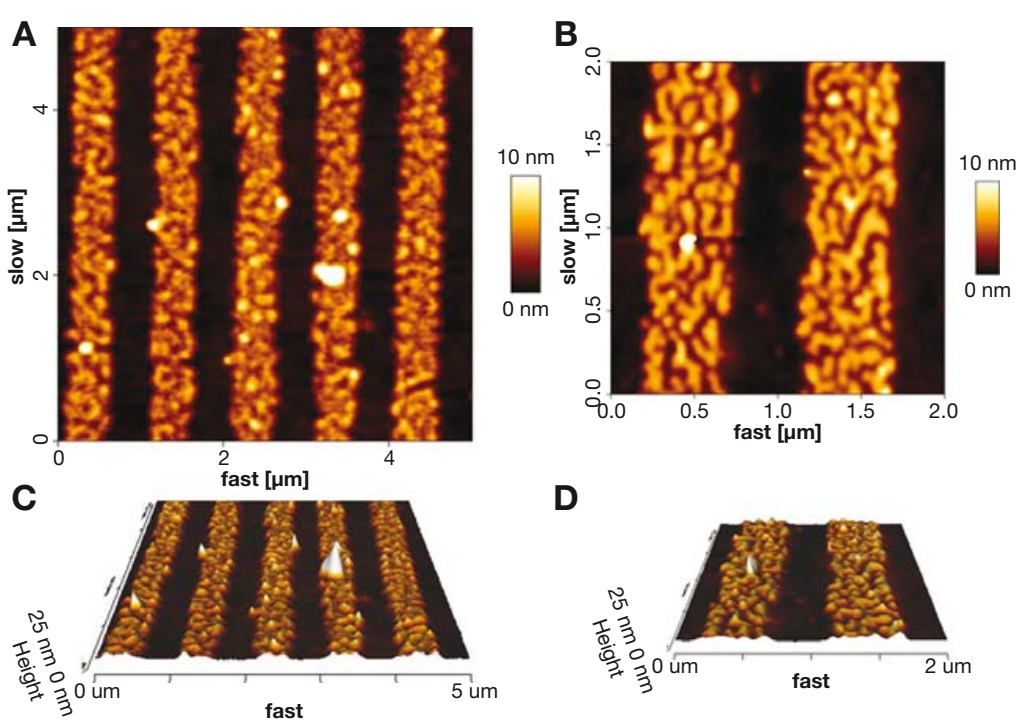

D

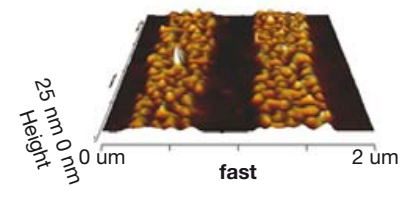

Fig. 4.

Atomic force microscopy images of a nanoscale periodic grating of proteins (antibodies) fabricated by $\mu \mathrm{CP}$ used for optical label-free detection of an immunoassay. The period of the grating is $1 \mu \mathrm{m}$, and the linewidth is $500 \mathrm{~nm}$

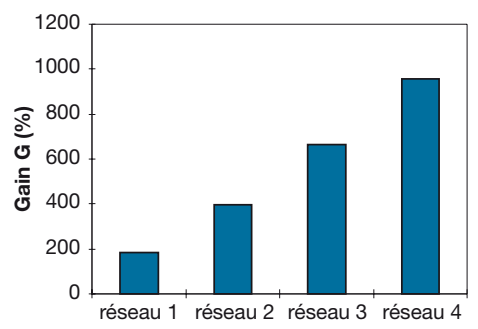

Fig. 5.

Results of protein A/anti-protein A antibody interactions detected by label-free optical detection. On the left side, the positive specific interaction leads to a positive diffraction gain $G$ defined in the text. On the right side, the negative control experiment leads to a negative diffraction gain $G$. The different bars represent different arrays printed by $\mu C P$ of streptavidin molecules with different molecular surface density

validation, we have printed by $\mu \mathrm{CP}$ molecular gratings made of streptavidin (grating size: $400 \mu \mathrm{m} \times$ $400 \mu \mathrm{m}$, pitch: $1 \mu \mathrm{m}$, linewidth: $500 \mathrm{~nm})$. We then incubate the biotinylated protein A, acting as probe molecules, which are strongly attached to the surface of the chip through the very robust biotin/streptavidin linkage. After rinsing with PBS, we incubate the anti-protein A antibodies $(60 \mu \mathrm{g} / \mathrm{mL})$ that specifically interact with the protein $A$ and rinse with PBS. In a control experiment, the anti-protein $A$ antibodies were incubated on the surface directly after streptavidin printing in the absence of any probe molecules at the surface. We compare the signal before the antibody incuba-

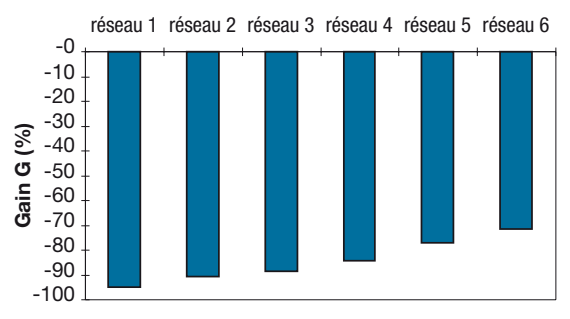

the intensity fluctuations of the incident light source. The second one named the gain, $G$, is intended to quantify the biological interaction. We define it as the increase of the ratio expressed here above after an interaction $\left(\frac{P_{1}}{P_{i}}\right)_{\text {after }}$ with respect to the ratio of the relative powers before the interaction $\left(\frac{P_{1}}{P_{\mathrm{i}}}\right)_{\text {before }}$ as

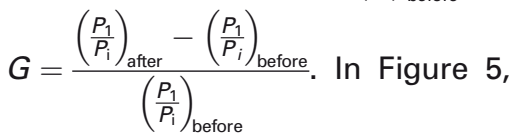
we observe clearly that in the case of a positive test (left side) where adsorption of the target molecules occurs on top of the printed lines due to specific probe/target interaction, the gain is strongly positive $(G=$ 200-800\%), whereas for the negative control experiment where the adsorption of the antibodies is unspecific, the gain is strongly negative (-80 to $90 \%)$. The forces of this detection method are that no labeling of the target molecules is required, the response is very strong and by just looking at the sign of the gain we can distinguish easily specific interaction events which are spatially correlated to the pitch of our initial grating and thus increase the diffraction efficiency $(G>0)$ from unspecific random surface interactions, spatially uncorrelated which reduces diffraction intensity $(G<0)$.

\section{Detection limit of the method and simulations}

tion and after rinsing. We demonstrate that the diffraction signal increases significantly (20-100\%) because of antigen/antibody interaction, whereas for the negative control experiment, the diffraction signal decreases because of nonspecific adsorption of antibodies on the surface that reduce the diffractive efficiency of the streptavidin grating. Results are shown in Figure 5. For quantification purposes, we manipulate two measurable parameters. The first one estimates the "efficiency" of the diffractive grating. It compares the diffracted power at order $1, P_{1}$, to the incident power $P_{\mathrm{i}}$ (or a fraction of $P_{\mathrm{i}}$ ). It is defined as the ratio $\frac{P_{1}}{P_{\mathrm{i}}}$, which presents the advantage of being insensitive to
A full simulation of the diffractive behavior of these molecular gratings upon biomolecular interactions has been performed. It is far beyond the scope of this article to detail these simulations. Our diffractive grating of lines is initially modeled by a grating of source bands that emit different complex light amplitudes. These light sources emit electromagnetic waves that "interfere" in the far-field. The model developed permits to analyze all the influences on the two previously defined parameters: the "efficiency" and the "gain." This model has been applied to the study of ideal situations of detection such as the trapping of targets by grating 
of probe lines: on the lines, on and at the edges of the lines, and inbetween the lines. The simulations show that biodetection by diffraction is a performing method that permits to differentiate, in one single step, just by evaluating the sign of the diffractive gain, specific and nonspecific interactions as observed in our experiments. Extensive simulations have then been performed with the aim of analyzing the influence of all the parameters both on the value of the diffractive signal and the gain. We propose pertinent ways to choose the controllable experimental parameters: normal incidence, wavelength as short as possible, small period but slightly superior to the wavelength, dimension of the gratings superior to four times the period, detector with a large area adjustable in position, and linewidth near $/ / p=0.5$. In typical experiments we have performed, with a pitch of $1 \mu \mathrm{m}$, the detection limit is around a few fM.

\section{Conclusion}

In this article, we have described a new technology for fabricating highly sensitive low-cost biochips that involves biopatterning of probe molecules at the nanoscale. The combination of low-cost patterning at the nanoscale and label-free optical detection allows us to propose a complete biochip platform, commercially available in a very near future. By reducing the cost of molecular analysis, we hope to penetrate the market of medical analysis because the proposed technology should permit to have access to sophisticated molecular analysis at an affordable cost. The validation of the technology for the detection of potential cancer biomarkers (Rho A proteins) is currently under investigation in collaboration with Institut Claudius Regaud in Toulouse within the framework of ONCOMATE project funded by InnaBiosante fundation.

\section{Conflict of Interest Statement:}

The authors declare no competing financial interests.

\section{References}

1. Arenkov $P$, Kuhktin $A$, Gemmel $A$, Voloshchuck S, Chupeeva V, Mirzabekov A (2000) Protein microchips: Use for immunoassay and enzymatic reactions. Anal Biochem 278: 12-131

2. Bernard $A$, Fitzli $D$, Sonderegger $P$, Delamarche E, Michel B, Bosshard HR, Biebuyck H (2001) Affinity capture of proteins from solution and their dissociation by contact printing. Nat Biotechnol 19: 866-9

3. Biebuyck H, Larsen A, Delamarche E, Michel B (1997) Lithography beyond light: microcontact printing with monolayer resists. IBM J Res Dev 41: 159-70

4. Ferrari M (2005) Cancer nanotechnology: Opportunities and challenges. Nat Rev 5: 161

5. Fodor SPA, Read JL, Pirrung M, Stryer L, Lu AT, Solas D (1991) Light-directed, spatially addressable parallel chemical synthesis. Science 251: 767-73
6. Gates B, Xu O, Stewart M, Ryan D, Willson CG, Whitesides GM (2005) New approaches to nanofabrication: molding, printing, and other techniques. Chem Rev 105: 1171-96

7. Kumar A, Whitesides GM (1993) Features of gold having micrometer to centimeter dimensions can be formed through a combination of stamping with an elastomeric stamp and an alkanethiol ink followed by chemical etching. Appl Phys Lett 63: 2002-4

8. Lalo H, Cau JC, Thibault C, Marsaud N, Severac C, Vieu C (2009) Microscale multiple biomolecules printing in one step using a PDMS macrostamp Microelectron Eng 86: 1428

9. Renault JP, Bernard A, Bietsch A, Michel B, Bosshard HR, Delamarche E, Kreiter M, Hecht B, Wild UP (2003) Fabricating arrays of single protein molecules on glass using microcontact printing. J Phys Chem B 107: 703-11

10. Schena M, Shalo D, Davis R, Brown $P$ (1995) Quantitative monitoring of gene expression patterns with a complementary DNA microarray. Science 270: 467-70

11. Souteyrand E (1999) DNA Chips: from elaboration to application. Analysis 27: 639-46

12. Thibault C, Leberre V, Casimirius $S$, Trévisiol E, François JM, Vieu C (2005) Direct microcontact printing of oligonucleotides for biochip applications. J Nanobiotechnol 3: 7(http://www.jnanobiotechnology.com/qc/content/3/1/7)

13. Thibault C, Severac C, Trévisiol E, Vieu C (2006) Microtransfer molding of hydrophobic dendrimers. Microelectron Eng 83: 1513

14. Vieu C, Carcenac F, Pépin A, Chen $Y$, Mejias M, Lebib A, Manin-Ferlazzo L, Couraud L, Launois H (2000) Electron beam lithography : resolution limits and applications. Appl Surf Sci 164: 111

15. Younan X, Whitesides GM (1998) Soft Lithography. Angew Chem Int Ed Engl 37: 550-75 